\title{
Fluid/gravity correspondence and the CFM black brane solutions
}

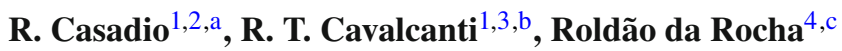 \\ ${ }^{1}$ Dipartimento di Fisica e Astronomia, Università di Bologna, via Irnerio 46, 40126 Bologna, Italy \\ 2 I.N.F.N., Sezione di Bologna, via B. Pichat 6/2, 40127 Bologna, Italy \\ ${ }^{3}$ Centro de Ciências Naturais e Humanas, Universidade Federal do ABC-UFABC, Santo André 09210-580, Brazil \\ ${ }^{4}$ Centro de Matemática, Computação e Cognição, Universidade Federal do ABC-UFABC, Santo André 09210-580, Brazil
}

Received: 25 July 2016 / Accepted: 30 September 2016 / Published online: 17 October 2016

(c) The Author(s) 2016. This article is published with open access at Springerlink.com

\begin{abstract}
We consider the lower bound for the shear viscosity-to-entropy density ratio, obtained from the fluid/ gravity correspondence, in order to constrain the postNewtonian parameter of brane-world metrics. In particular, we analyse the Casadio-Fabbri-Mazzacurati (CFM) effective solutions for the gravity side of the correspondence and argue that including higher-order terms in the hydrodynamic expansion can lead to a full agreement with the experimental bounds, for the Eddington-Robertson-Schiff post-Newtonian parameter in the CFM metrics. This lends further support to the physical relevance of the viscosityto-entropy ratio lower bound and fluid/gravity correspondence. Hence we show that CFM black branes are, effectively, Schwarzschild black branes.
\end{abstract}

\section{Introduction}

Black-hole solutions of the Einstein equations in general relativity (GR) are useful tools to investigate the spacetime structure and quantum effects in any possible theory of gravity. In particular, models with extra spatial dimensions are prominent candidates as extensions of Einstein GR [1], leading to important consequences, not only for black-hole physics [24], but also for particle physics, cosmology, as well as for the astrophysics of supermassive objects [5]. In this context, black strings play a notable role in the quest for physically viable and realistic models [6-8].

Fluid dynamics is an effective description of interacting quantum field theories (QFTs) at long wavelengths [9]. As a low-energy effective field theory, fluid dynamics is determined by a derivative expansion of the local fluid variables, and is used to describe near equilibrium systems. The deriva-

\footnotetext{
a e-mail: casadio@bo.infn.it

be-mail: rogerio.cavalcanti@ufabc.edu.br

c e-mail: roldao.rocha@ufabc.edu.br
}

tive expansion is determined by the symmetries of the system and its thermodynamical features. Transport coefficients, such as viscosities and conductivities, assess how perturbations propagate under relaxation and, besides being theoretically predictable, they can be measured experimentally.

The AdS/CFT correspondence, which relates the coupled dynamics of $S U(N)$ gauge theories to gravity in antide Sitter (AdS) spaces [10-13], has been used to connect those two realms of modern physics, namely gravity and QFT. It holographically relates a strongly interacting QFT at large $N$ with the dynamics provided by gravity in asymptotically AdS spacetime. In the long-wavelength limit, the fluid/gravity correspondence has been recently proposed as a framework related to the AdS/CFT correspondence [1416], mapping black holes in asymptotically AdS spacetimes to the fluid dynamics of a strongly coupled boundary field theory. One of the most remarkable predictions of the AdS/CFT and fluid/gravity correspondence is the ratio of the shear viscosity-to-entropy density, which is universal for a large class of strongly coupled (gauge theory) isotropic plasmas [9]. The universality of this ratio plays a prominent role for gauge theories that are dual to gravitational backgrounds [14-20]. Moreover, the same results were obtained by employing the Green-Kubo formula in Ref. [21].

On the other hand, black strings present hydrodynamic features such as viscosity, diffusion rates, diffusion constants and other transport coefficients [22], besides temperature and entropy. From the perspective of the holographic principle [10], a black string corresponds to a certain finitetemperature QFT in lower dimensions. In this context, the hydrodynamic features of the horizon of a black string can be identified with the hydrodynamic behaviour of a dual theory. For such QFTs, the ratio of the shear viscosity $\eta$ to entropy density $s$ is bounded by the universal value [17]

$$
\frac{\eta}{s} \gtrsim \frac{\hbar}{4 \pi k_{\mathrm{B}}} \simeq 6.08 \times 10^{-13} \mathrm{Ks}
$$


for an extended range of thermal QFTs. The above inequality holds for all known substances, including, for example, liquid helium, water, and the quark-gluon plasma, produced at the relativistic heavy ion collider (RHIC). Therefore, it has been conjectured to represent a universal lower bound for all materials, ${ }^{1}$

$\frac{\eta}{s}=\frac{1}{4 \pi}$

which is called the Kovtun-Starinets-Son (KSS) bound [16, 17]. In the case of Einstein gravity, the ratio $\eta / s$ equals $1 / 4 \pi$. This bound is obtained in bulk supergravity from a recent calculation in $\mathcal{N}=4$ supersymmetric $S U\left(N_{c}\right)$ Yang-Mills theories, in the regime of infinite $N_{c}$ and large 't Hooft coupling $g^{2} N_{c}[18]$, yielding

$\frac{\eta}{s}=\frac{1}{4 \pi}\left[1+\frac{135 \zeta(3)}{8\left(2 g^{2} N_{c}\right)^{3 / 2}}+\cdots\right]$,

where $\zeta(3)$ is the Apéry constant, and the next-to-leading term represents the first string theory correction to GR. The KSS bound is a powerful tool for studying strongly interacting systems, like the quark-gluon plasma, and it is further employed to analyse trapped atomic gases. Strongly interacting Fermi gases of atoms have been noticed to be ruled by hydrodynamics [23], presenting finite shear viscosity at finite temperature. The bound (2) holds in relativistic QFTs at finite temperature and chemical potential [16,17], for at least a single component non-relativistic gas of particles with either spin zero or spin 1/2 [24]. The entropy of the dual QFT equals the entropy of a black string, which is proportional to the area $A$ (volume) of its event horizon in Planck units, $S=A / 4 G_{5}$, where $G_{5}$ is the five-dimensional gravitational constant.

According to the AdS/CFT duality, any given asymptotically AdS bulk geometry should be equivalent to specific states of the gauge theory on the boundary. In fact, the AdS bulk geometry can be mapped into vacuum states of the gauge theory, whereas AdS-Schwarzschild black holes are related to thermal states of the corresponding gauge theory on the boundary. Hence, a fluid can be associated with the bulk black-hole solution. Moreover, the bulk dynamics is governed by Einstein's equations, with the AdS-Schwarzschild black hole as a typical solution [25]. Each different solution is equivalent to a thermal state, corresponding to black strings in AdS [26]. The dynamical framework can be realised by a system in local thermodynamical equilibrium, corresponding to a non-uniform black string in AdS, which evolves according to effective hydrodynamical equations corresponding to

\footnotetext{
${ }^{1}$ We shall mostly use units with $\hbar=c=k_{\mathrm{B}}=1$, four-dimensional indices will be represented by Greek letters, $\mu=0, \ldots, 3$ and fivedimensional indices by capital Latin letters, $A=0, \ldots, 4$.
}

the Einstein equations [25]. Such a system will subsequently relax towards a global equilibrium state, corresponding to a uniform black string in AdS [26].

Motivated by the above picture, we shall here explore the fluid/gravity duality for the five-dimensional black string [5, 27,28] associated with the effective four-dimensional Casadio-Fabbri-Mazzacurati (CFM) solutions [29]. CFM metrics generalise the Schwarzschild solution and have a parametrised post-Newtonian (PPN) form with parameter $\beta$, which is the usual Eddington-Robertson-Schiff parameter used to describe the classical tests of GR. ${ }^{2}$ This parameter allows for a direct comparison with experimental data in the weak-field limit, which is accurate enough to include most solar system tests [30]. In general, one can construct a dual fluid stress tensor by solving the bulk Einstein equations in asymptotically AdS spacetime and taking the longwavelength limit, but there are only a few black-hole solutions that admit such a hydrodynamic dual on the boundary, even asymptotically. The prototype is the Schwarzschild metric, however, the CFM solutions can also be obtained as fourdimensional vacuum brane solutions of Einstein gravity with a cosmological constant. In a second-order derivative theory of five-dimensional gravity interacting with other fields in AdS, for instance type II B supergravity in $\mathrm{AdS}_{5} \times S^{5}$, the CFM solutions can be realised as universal sub-sectors, regarding pure gravity with negative cosmological constant, in the long-wavelength limit.

On the gravity side, we shall thus consider a fivedimensional CFM black string, associated with a fourdimensional CFM black hole, and the four-dimensional dual fluid description will necessarily carry an imprint of the parameter $\beta$. The fluid/gravity correspondence will then be shown to provide a bound for $\beta$, corresponding to the universal KSS bound (2). In particular, both the shear viscosity and the black string entropy will depend on $\beta$, and Eq. (2) will read

$\frac{\eta(\beta)}{s(\beta)} \gtrsim \frac{1}{4 \pi}$

which is precisely what gives rise to the bound on $\beta$. We shall also argue that this bound can be made to agree with observations, provided higher-order terms are included in the hydrodynamic expansion of the stress tensor.

This paper is organised as follows: in Sect. 2 the CFM solutions are reviewed; in Sect. 3, the Green-Kubo formula is used to bound the PPN parameter through the shear viscosityto-entropy density ratio in the linear regime, and we show how the estimates improve by including higher-order hydro-

\footnotetext{
$\overline{2}$ As usual [1] the four-dimensional brane-world black hole is viewed as a transverse section of the five-dimensional black string, producing real world post-Newtonian corrections.
} 
dynamical terms in Sect. 4; finally we discuss our results in Sect. 5.

\section{Casadio-Fabbri-Mazzacurati brane-world solutions}

Solutions of the Einstein field equations on the brane are not uniquely determined by the matter energy density and pressure, since gravity can propagate into the bulk and generates a Weyl term onto the brane itself. Taking that into account, the CFM metrics [27] are vacuum brane solutions, like the tidally charge metric in Ref. [31], and contain a PPN parameter $\beta$, which can be measured on the brane. The case $\beta=1$ corresponds to the exact Schwarzschild solution on the brane, and it extends to a homogeneous black string in the bulk. Furthermore, it was observed in Ref. [29] that $\beta \approx 1$, in solar system measurements. More precisely, the deflection of light in the classical tests of GR provides the bound $|\beta-1| \lesssim 0.003$ [30]. The parameter $\beta$ also measures the difference between the inertial mass and the gravitational mass of a test body, besides affecting the perihelion shift and describing the Nordtvedt effect [29]. Finally, measuring $\beta$ provides information regarding the vacuum energy of the brane-world or, equivalently, the cosmological constant $[1,29]$.

We recall the effective four-dimensional Einstein equations on the brane can be expressed as

$$
\begin{aligned}
G_{\mu \nu}= & 8 \pi G_{\mathrm{N}} T_{\mu \nu}-\frac{\Lambda_{4}}{2} g_{\mu \nu}+\frac{\kappa_{5}^{4}}{4}\left[\frac{g_{\mu \nu}}{2}\left(T^{2}-T_{\alpha \beta} T^{\alpha \beta}\right)\right. \\
& \left.+T T_{\mu \nu}-T_{\mu \alpha} T_{\nu}^{\alpha}\right]-\mathcal{E}_{\mu \nu}
\end{aligned}
$$

where $\kappa_{5}^{2}=8 \pi G_{5}$ and $G_{\mathrm{N}}=\kappa_{5}^{2} \sigma / 48 \pi$ ( $\sigma$ being the brane tension), $T_{\mu \nu}$ denotes the energy-momentum tensor of brane matter, $T \equiv T_{\mu}^{\mu}$, and $\mathcal{E}_{\mu \nu}$ is the Weyl tensor term. For brane vacuum, $T_{\mu \nu}=0$, and absorbing the bulk cosmological constant $\Lambda_{4}$ into the bulk warp factor, the above field equations reduce to

$R_{\mu \nu}=-\mathcal{E}_{\mu \nu}$.

We are in particular interested in static spherically symmetric systems on the brane, whose general metric can be written as

$g_{\mu \nu} \mathrm{d} x^{\mu} \mathrm{d} x^{\nu}=-N(r) \mathrm{d} t^{2}+B(r) \mathrm{d} r^{2}+r^{2} \mathrm{~d} \Omega^{2}$.

The CFM metrics are obtained by relaxing the condition $N(r)=B^{-1}(r)$, valid, for example, for the Schwarzschild and Reissner-Nordström metrics. In fact, for black strings, such a condition will result in a central singularity extending all along the extra dimension and a singular bulk horizon [29], a configuration which, moreover, suffers from the well- known Gregory-Laflamme instability [32]. The CFM solution I is obtained, by fixing $N(r)$ equal to the Schwarzschild form and then determining $B(r)$ from the field equations (6), whereas the CFM solution II follows from the same procedure but starting from a metric coefficient $N(r)$ of the Reissner-Nordström form [31].

\subsection{CFM solution I}

For the first case, the metric coefficients in Eq. (7) are given by

$$
\begin{aligned}
& N_{I}(r)=1-\frac{2 G_{\mathrm{N}} M}{r} \text { and } \\
& B_{I}(r)=\frac{1-\frac{3 G_{\mathrm{N}} M}{2 r}}{\left(1-\frac{2 G_{\mathrm{N}} M}{r}\right)\left[1-\frac{G_{\mathrm{N}} M}{2 r}(4 \beta-1)\right]} \equiv B(r) .
\end{aligned}
$$

The solution (8) depends upon just one parameter $\beta$ and the Minkowski vacuum is recovered for $M \rightarrow 0$. The horizon radius $r=R$ on the brane is then determined by the algebraic equation $1 / B(R)=0$, and this black hole is either hotter or colder than the Schwarzschild black hole of equal mass $M$ depending upon the sign of $(\beta-1)$ [29]. For example, assuming $\beta=5 / 4$, one finds two solutions equal to the Schwarzschild radius $R_{S}=2 G_{\mathrm{N}} M$. Note also that the four-dimensional Kretschmann scalar $K^{(I)}=R_{\mu \nu \rho \sigma} R^{\mu \nu \rho \sigma}$ diverges for $r=0$ and $r=3 G_{\mathrm{N}} M / 2<R_{S}$.

\subsection{CFM solution II}

The second solution for the metric coefficients reads

$$
\begin{aligned}
& N_{I I}(r)=1-\frac{2 G_{\mathrm{N}} M}{r}+\frac{2 G_{\mathrm{N}}^{2} M^{2}}{r^{2}}(\beta-1) \text { and } \\
& B_{I I}(r)=B(r) .
\end{aligned}
$$

In this case the classical radius $R$ for the black-hole horizon is given by $R=R_{S}$ and $R=R_{S}(\beta-1 / 4)$. Moreover, the Kretschmann scalar diverges at each black-hole horizon [5], hence indicating a physical singularity.

A comprehensive analysis of the causal structure and further features on both CFM solutions can be found in Refs. [5,27,29].

\section{Bounding the PPN parameter in the linear regime}

As previously mentioned, hydrodynamics can be viewed as a low-energy effective description for the low-momentum regime of correlation functions. We are particularly interested in the Green-Kubo formula in the linear response theory, where an important fluid transport coefficient, the shear viscosity, arises in response to a perturbation in the fluid 
stress-energy tensor. The Kubo formula can further relate the shear viscosity to the absorption cross section of low-energy gravitons [19]. ${ }^{3}$

Let us consider an action $S$, and introduce a set of sources $J^{a}$ coupled to a set of operators $O^{a}$, namely, $S \mapsto S+$ $\int \mathrm{d}^{4} x J_{a}(x) O^{a}(x)$, where $x^{\mu}=\left(x^{0}, x^{i}\right)$. If the spacetime is flat and the vacuum expectation values (VEV) of all the $O^{a}$ vanish in the absence of the sources $J_{a}$, one has

$\left\langle O^{a}(x)\right\rangle=-\int \mathrm{d} y G_{R}^{a \mid b}(x ; y) J_{b}(y)$,

where $G_{R}(x ; y)=G_{R}(x-y)$ is the retarded Green function of $O^{a}$ in position space given by

$i G_{R}^{a \mid b}(x ; y)=\theta\left(x^{0}-y^{0}\right)\left\langle\left[O^{a}(x), O^{b}(y)\right]\right\rangle$.

Using the interaction picture in QFT [13], one can immediately see that the variation

$\delta\left\langle O^{a}(q)\right\rangle=-G_{R}^{a \mid b}(q) J_{b}(q)$,

where $G_{R}(q)$ denotes the retarded Green function in momentum space with $q^{\mu}=(\omega, k)$. This simple linear formalism can be generalised to curved spacetimes and non-vanishing $\mathrm{VEV}$ in order to describe a fluid in the conditions of interest here.

A common way to derive the Kubo formula is, in fact, to couple four-dimensional gravity $g_{\mu \nu}$ to the fluid and determine how the fluid energy-momentum tensor responds to gravitational perturbations. ${ }^{4}$ In particular, for small metric perturbations which vary slowly in space and time, $g_{\mu \nu}=$ $\bar{g}_{\mu \nu}+h_{\mu \nu}$ with $\left\|h_{\mu \nu}\right\| \ll 1$, the stress tensor can be doubly expanded in the metric fluctuation $h_{\mu \nu}$ and in gradients [33]. This procedure yields a generalisation of Eq. (10) to higher orders for the operator $T_{\mu \nu}$, namely

$$
\begin{aligned}
\left\langle T^{\mu \nu}(x)\right\rangle= & \left\langle T^{\mu \nu}\right\rangle_{h=0}-\frac{1}{2} \int \mathrm{d}^{4} y G_{\mathrm{R}}^{\mu \nu \mid \rho \sigma}(x ; y) h_{\rho \sigma}(y) \\
& +\frac{1}{8} \int \mathrm{d}^{4} y \int \mathrm{d}^{4} z G_{\mathrm{R}}^{\mu \nu|\rho \sigma| \tau \zeta}(x ; y, z) h_{\rho \sigma}(y) h_{\tau \zeta}(z)+\cdots \\
\equiv & \left\langle T_{(0)}^{\mu \nu}\right\rangle+\left\langle T_{(1)}^{\mu \nu}\right\rangle+\left\langle T_{(2)}^{\mu \nu}\right\rangle+\cdots,
\end{aligned}
$$

where $G_{R}^{\mu \nu \mid \cdots}$ are retarded $n$-point correlators, with the measurement point $z$ having the largest time, and we also assume the metric fluctuations $h_{\rho \sigma}$ vanish in the far past. The fluid response is then obtained from the stress tensor conservation law $\nabla_{\mu} T^{\mu \nu}=0$, together with the condition that the fluid describes a conformal theory, namely, $T_{\mu}^{\mu}=0$. We shall here analyse the first-order formalism, and illustrate how our results can be further refined by considering second-order terms in the next section.

\footnotetext{
${ }^{3}$ Some equivalent approaches can be found, e.g., in Refs. [14,16].

${ }^{4}$ Note that, although the spacetime is flat in our laboratories, the effect of spacetime curvature on hydrodynamics becomes extremely relevant in astrophysics.
}

At zeroth order in the derivatives, the energy-momentum tensor reads

$T_{(0)}^{\mu \nu}=(\epsilon+P) u^{\mu} u^{v}+P \bar{g}^{\mu \nu}$,

where $u^{\mu}$ is the fluid four-velocity, $\epsilon$ denotes its energy density, $P$ the pressure and $\bar{g}_{\mu \nu}$ is again the unperturbed metric on the four-dimensional boundary where the fluid lives. ${ }^{5}$

Since in the linear response theory the metric can be seen as the source of the stress-energy tensor, the response of the two-point function of the associated perturbed metric can then be written as the first-order term $\left\langle T_{\mu \nu}^{(1)}\right\rangle$ in the expansion (13) [34],

$\left\langle T^{\mu v}(x)\right\rangle \sim \int \mathrm{d} y G_{R}^{\mu \nu \mid \alpha \beta}(x ; y) h_{\alpha \beta}(y)$,

where the retarded Green function is $G_{R}^{\mu \nu \mid \alpha \beta}=\left\langle T^{\mu \nu}(x)\right.$ $\left.T^{\alpha \beta}(y)\right\rangle$. In the gauge/gravity duality, the metric is dual to the stress-energy tensor [12]. Hence, from the gravity point of view of the correspondence, the above perturbation should arise from metric fluctuations $h_{\mu \nu}$ of the appropriate black brane metric.

The first-order expansion (in derivatives) of the hydrodynamical stress-energy tensor, also known as the constitutive equation, includes dissipative terms, as well as shear and bulk viscosities. In curved spacetimes, the first-order contribution is given by [35]

$$
\begin{aligned}
T_{(1)}^{\mu \nu}= & -P^{\mu \alpha} P^{\nu \beta}\left[\eta\left(\nabla_{\alpha} u_{\beta}+\nabla_{\beta} u_{\alpha}-\frac{2}{3} \bar{g}_{\alpha \beta} \nabla_{\lambda} u^{\lambda}\right)\right. \\
& \left.+\zeta \bar{g}_{\alpha \beta} \nabla_{\lambda} u^{\lambda}\right]
\end{aligned}
$$

where $\eta$ is the shear viscosity, $\zeta$ the bulk viscosity, $\nabla_{\mu}$ represents the covariant derivative in the (generally curved) spacetime metric $\bar{g}_{\mu \nu}$. In addition, $P^{\mu \nu}=\bar{g}^{\mu \nu}+u^{\mu} u^{\nu}$ is the projection tensor along spatial directions, which enables us to express the constitutive equation in a covariant manner.

We shall next restrict ourselves to a particular type of perturbations which is simpler to treat using the AdS/CFT correspondence. In fact, we suppose, as usual, that the contribution to the shear viscosity $\eta$ comes only from the component $h_{12}=h_{12}(t)$ of $h_{\mu \nu}$, with all other $h_{\mu \nu}=0[13,34]$. Since we assumed such fluctuations around thermal equilibrium are small, we can say the fluid has uniform temperature $\mathcal{T}\left(x^{\mu}\right)=\mathcal{T}_{0}$ and is at rest in the chosen frame, that is, $u^{\mu}=\left(1, u^{i}=0\right)$. Moreover, this perturbation has spin-2 with respect to the spatial $S O(3)$ group and it cannot excite linear-order fluctuations of the velocity (which is a vector) or of the temperature (a scalar). Therefore, $u^{i}=0$ and $\mathcal{T}=\mathcal{T}_{0}$ remain valid up to linear order.

\footnotetext{
5 Minkowski spacetime is just a particular case corresponding to a Schwarzschild-type black brane in the bulk.
} 
The shear viscosity of the dual theory can be computed from gravity in a number of equivalent approaches [13,14, 16]. We shall employ the Kubo formula, which relates the viscosity to equilibrium correlation functions, in order to compute the $T^{12}$ component of the stress-energy tensor, in a large distance and long time scale regime. The non-vanishing contribution in the covariant derivative arises from the Christoffel symbol

$\nabla_{1} u_{2}=\partial_{1} u_{2}-\Gamma_{12}^{\alpha} u_{\alpha}=-\Gamma_{12}^{0} u_{0}=-\frac{1}{2} \partial_{0} h_{12}$.

The component $\nabla_{2} u_{1}$ is obtained analogously and the other components vanish. Hence, only the first two terms inside the brackets in Eq. (16) contribute to $T_{(1)}^{\mu \nu}$, and we have

$\delta\left\langle T_{(1) 12}\right\rangle \sim-\eta\left(\nabla_{1} u_{2}+\nabla_{2} u_{1}\right)=-\eta \partial_{0} h_{12}$.

By taking the Fourier transform of Eq. (18), one obtains [34]

$\delta\left\langle T_{(1) 12}(\omega, k=0)\right\rangle=i \omega \eta h_{12}$.

A perturbed fluid Lagrangian is correspondingly given by $\delta \mathcal{L}=h_{\mu \nu}\left(x^{0}\right) T^{\mu \nu}\left(x^{\alpha}\right)=h_{12}\left(x^{0}\right) T^{12}\left(x^{\alpha}\right)$, for which Eq. (12) reads

$\delta\left\langle T^{12}\right\rangle=-G_{R}^{12 \mid 12}(q) h_{12}$,

where $G_{R}^{12 \mid 12}(q)=-i \int \mathrm{d}^{4} x e^{-i q_{\mu} x^{\mu}} \theta\left(x^{0}\right)\left\langle T^{12}\left(x^{\mu}\right) T^{12}\right.$ (0) $\rangle$. Equation (19) represents the same linear response relation as Eq. (20), and the Green-Kubo formula can therefore be obtained [13,34]:

$\eta=-\lim _{\omega \rightarrow 0} \frac{\mathfrak{\Im} G_{R}^{12 \mid 12}(\omega, 0)}{\omega}$,

where $\mathfrak{\Im}$ denotes the imaginary part.

Our first concern here is to provide analytic expressions for asymptotically AdS black strings which solve the full five-dimensional Einstein equations. The CFM black strings are "tubular", codimension one, black branes in AdS [5], corresponding to a late time behaviour of their dual fluid. Two families of such solutions are obtained from the same assumption that leads to the CFM brane metrics reviewed in Sect. 2, namely by relaxing the condition that the metric coefficient $g_{t t}$ is (minus) the inverse of the metric coefficient $g_{r r}$. These two families of black strings are likewise expressed in terms of the PPN parameter $\beta$ [29], and reduce to the five-dimensional Schwarzschild black string solution, when $\beta \rightarrow 1$. CFM black branes in AdS can also be obtained in a perturbative approach of CFM black holes on the brane [5]. Our five-dimensional metrics are, in particular, given by

$g_{A B} \mathrm{~d} x^{A} \mathrm{~d} x^{B}=-N(r) \mathrm{d} t^{2}+B(r) \mathrm{d} r^{2}+r^{2} \mathrm{~d} \Omega^{2}$, where $\mathrm{d} \Omega^{2}=\ell^{-2} \mathrm{~d} \vec{x}^{2}=\ell^{-2}\left(\mathrm{~d} x_{1}^{2}+\mathrm{d} x_{2}^{2}+\mathrm{d} x_{3}^{2}\right)$, the length $\ell$ is related to the AdS curvature $[26,36]$ and the metric coefficients $N$ and $B$ equal the CFM expressions (8) or (9). The entropy of these black strings can be computed by using the Hawking-Bekenstein formula [16],

$S=\frac{A}{4 G_{5}}=\frac{R^{3} V_{3}}{4 G_{5} \ell^{3}}$,

where $R$ is the horizon radius determined by $1 / B(R)=0$ and $V_{3}=\ell \int \mathrm{d} \Omega$. Moreover, the volume density of entropy $s=$ $S / V_{3}$ must be finite, which requires that $\beta<5 / 4$ in Eqs. (8) and (9). It is also convenient to trade the radial coordinate $r$ for the dimensionless $u=R / r$, and define the length $\bar{M}=$ $G_{\mathrm{N}} M$, so that

$B(u)=\frac{1-\frac{3 \bar{M}}{2 R} u}{\left(1-\frac{2 \bar{M}}{R} u\right)\left[1-\frac{\bar{M}}{2 R} u(4 \beta-1)\right]}$

and the metric finally reads

$$
\begin{aligned}
g_{A B} \mathrm{~d} x^{A} \mathrm{~d} x^{B} & =-N(u) \mathrm{d} t^{2}+B(u) \frac{R^{2}}{u^{4}} \mathrm{~d} u^{2}+\frac{R^{2}}{u^{2}} \mathrm{~d} \Omega^{2} \\
& \equiv g_{u u} \mathrm{~d} u^{2}+g_{\mu \nu} \mathrm{d} x^{\mu} \mathrm{d} x^{\nu} .
\end{aligned}
$$

Our main aim is now to predict a value for $\beta$ exclusively from the KSS bound (2), implied by the fluid/gravity correspondence. The background metric (25) can be perturbed as $g_{A B} \mapsto g_{A B}+h_{A B}[14,17]$. As mentioned before, the contribution to the shear viscosity comes from a single component of the metric fluctuations. Denoting this term by $\phi=$ $\phi(t, u, \vec{x})$, the corresponding wave equation reads $[16,34]$

$\partial_{u}\left(\sqrt{-g} g^{u u} \partial_{u} \phi\right)+\sqrt{-g} g^{\mu \nu} \partial_{\mu} \partial_{\nu} \phi=0$.

By taking the Fourier transform along the time direction and disregarding the spatial momentum, $\phi \simeq e^{i \omega t} \Phi(u)$, one finds

$\partial_{u}\left(\sqrt{-g} g^{u u} \partial_{u} \Phi\right)-\sqrt{-g} g^{t t} \omega^{2} \Phi \simeq 0$,

which can be specialised for both CFM metrics I and II, respectively provided by Eqs. (8) and (9), that is,

$\frac{\mathrm{d}^{2} \Phi}{\mathrm{d} u^{2}}+\frac{V}{u} \frac{\mathrm{d} \Phi}{\mathrm{d} u}+\left(1-\frac{2 \bar{M}}{R} u\right) \omega^{2} \Phi=0$

where $V=V_{I, I I}$, with

$$
\begin{aligned}
V_{I}= & R\left[\frac{1}{(4 \beta-1) \bar{M} u-2 R}+\frac{1}{4 \bar{M} u-2 R}\right. \\
& \left.+\frac{1}{2 R-3 \bar{M} u}\right]+\frac{5}{2},
\end{aligned}
$$


Fig. 1 Allowed range for the PPN parameter $\beta$ as a function of the mass $M$ (in Solar mass units), for CFM I (left panel) and CFM II (right panel) in the linear regime
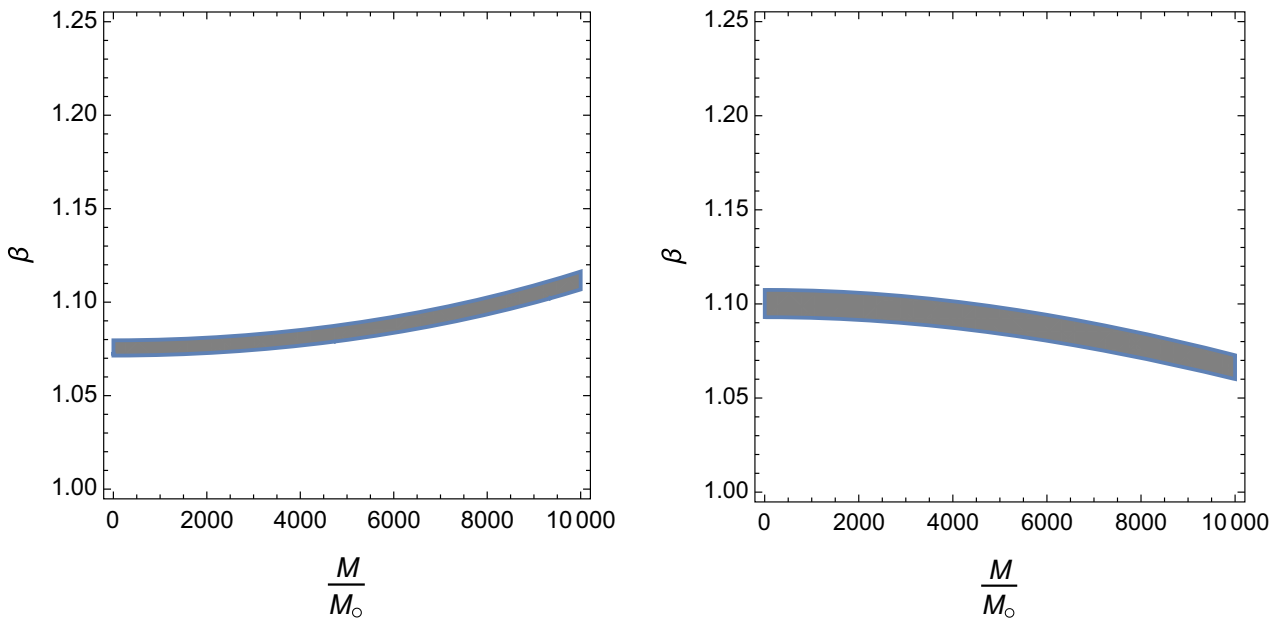

for the CFM case I and

$$
\begin{aligned}
V_{I I}= & R\left[\frac{1}{(4 \beta-1) \bar{M} u-3 R}+\frac{1}{4 \bar{M} u-2 R \beta}\right. \\
& \left.+\frac{1}{4 R-3 \bar{M} u}\right]+\frac{9}{2},
\end{aligned}
$$

for the CFM case II. Following the gauge/gravity prescription, we can then proceed to calculate the retarded Green function [14-16],

$G_{R}(\omega, \overrightarrow{0} ; \beta)=-\left.\sqrt{-g} g^{u u} \Phi^{*} \frac{\mathrm{d} \Phi}{\mathrm{d} u}\right|_{u \rightarrow 0}$,

for both cases in Eqs. (29) and (30), and then derive the shear viscosity from the Green-Kubo formula (21). A bound on the PPN parameter $\beta$ can finally be obtained from the KSS lower bound (2). From the propagator (31), by inserting Eq. (21) in Eq. (4), one in fact finds

$$
\frac{\eta(\beta)}{s(\beta)}=-\frac{1}{s(\beta)} \lim _{\omega \rightarrow 0} \frac{\Im G_{R}(\omega, k=0 ; \beta)}{\omega}=\frac{1}{4 \pi},
$$

where we remarked that both $s$ and $\eta$ do depend on $\beta$. However, the solutions to Eq. (31) are cumbersome linear combinations of integral exponentials and Hermite functions, and we shall therefore just display the results graphically, in the near horizon approximation.

The resulting bound for $\beta$ is displayed graphically in Fig. 1, where the grey bands correspond to the allowed range for varying black string mass $M$ in units of the solar mass $M_{\circ}$. The plot in the left panel is for the CFM solution I and $M_{\circ}<M<10^{4} M_{\circ}$. For $M=M_{\circ}$, the PPN parameter must lie in $1.0772 \lesssim \beta \lesssim 1.0803$, whereas $1.1074 \lesssim \beta \lesssim 1.1143$ for a brane-world CFM I black hole having a mass equivalent to $10^{4}$ solar masses. Similarly, for the CFM II black hole, we point out that if $M=M_{\circ}$, the bound on the shear viscosity-to-entropy ratio yields $1.0913 \lesssim \beta \lesssim 1.1093$, and $1.0602 \lesssim \beta \lesssim 1.0731$ for $M=10^{4} M_{\circ}$. On the other hand, the perihelion shift provides an observational value of $|\beta-1| \lesssim 0.003$ and the study of the Nordtvedt effect yields the bound $|\beta-1| \lesssim 0.00023$ [30]. Both experimental bounds fall outside the bands shown in Fig. 1, although they are close in value. Nevertheless, we shall argue in the next section how the situation can be significantly improved.

\section{Second-order improvements}

The results obtained in Sect. 3 are not far from the observational data, nevertheless they still appear to be ruled out by the experimental bounds. This could signal that the fluid/gravity correspondence fails for these systems, or that the approximations we employed are too strong. In particular, Eq. (32) was obtained in the linear hydrodynamics regime, corresponding to the first two terms in Eq. (13), that is, Eqs. (14) and (16). We can instead tackle the non-linear dynamics by keeping higher-order terms in the expansion (13) of the stress-energy tensor in velocity and gradients multiplied by the corresponding hydrodynamic coefficients [25]. In particular, we shall here include the second-order regime of a dual hydrodynamic prescription $[9,25,33,37,38]$, in order to refine the bounds on the PPN parameter $\beta$.

The second-order hydrodynamic analysis is rather involved $[9,25,33,37,39,40]$, so let us first introduce some compact notation. For any second rank tensor $O^{\alpha \beta}$, we denote $O^{\langle\alpha \beta\rangle} \equiv$ $\frac{1}{2} P^{\alpha \mu} P^{\beta \nu} O_{(\mu \nu)}-\frac{1}{3} P^{\alpha \beta} P^{\mu \nu} O_{\mu \nu}$, which is transverse to the fluid motion, $u_{\mu} O^{\langle\mu \nu\rangle}=0$, and traceless $g_{\mu \nu} O^{\langle\mu \nu\rangle}=0$. In addition, $\sigma^{\alpha \beta}=P^{\alpha \mu} P^{\beta \nu} \nabla_{(\mu} u_{v)}-\frac{2}{3} P^{\alpha \beta} P^{\mu \nu} \nabla_{\mu} u_{v}$ is the shear tensor and $\Omega^{\alpha \beta}=P^{\alpha \mu} P^{\beta \nu} \nabla_{[\mu} u_{\nu]}$ denotes the vorticity tensor, as they appear in the $T_{(1)}^{\mu \nu}$ of Eq. (16). We next express the derivatives along the fluid 4-velocity in terms of transverse derivatives by means of the zeroth-order equations of motion [33],

$u^{\rho} \nabla_{\rho} u^{\mu}=-\nabla_{\perp}^{\mu} \ln T, \quad u^{\rho} \nabla_{\rho} \ln T=-\frac{1}{2}\left(\nabla_{\perp} \cdot u\right)$, 
where $\nabla_{\perp}^{\mu} \equiv P^{\mu \alpha} \nabla_{\alpha}$ and $\nabla_{\perp} \cdot u=\nabla \cdot u \equiv \nabla_{\mu} u^{\mu}$. All possible contributions to the stress-energy tensor are $\sigma^{\mu \nu}(\nabla \cdot u)$, $\sigma_{\lambda}^{\langle\mu} \sigma^{\nu\rangle \lambda}, \quad \nabla^{\langle\mu} \ln T \nabla^{\nu\rangle} \ln T, \quad \sigma^{\langle\mu}{ }_{\lambda} \Omega^{\nu\rangle \lambda}, \quad \nabla^{\langle\mu} \nabla^{\nu\rangle} \ln T$, $u_{\alpha} R^{\alpha\langle\mu \nu\rangle \beta} u_{\beta}, \Omega^{\langle\mu} \Omega^{\nu\rangle \lambda}$, and $R^{\langle\mu \nu\rangle}$, where $R^{\alpha \beta \gamma \delta}$ is the Riemann tensor. But the only ones that transform homogeneously under Weyl transformations are [33]

$$
\begin{aligned}
& \mathcal{A}_{1}^{\mu \nu}=R^{\langle\mu \nu\rangle}-\left(\nabla^{\langle\mu} \nabla^{\nu\rangle} \ln T-\nabla^{\langle\mu} \ln T \nabla^{\nu\rangle} \ln T\right), \\
& \mathcal{A}_{2}^{\mu \nu}=R^{\langle\mu \nu\rangle}-u_{\alpha} u_{\beta} R^{\alpha\langle\mu \nu\rangle \beta}, \\
& \mathcal{A}_{3}^{\mu \nu}=\sigma_{\lambda}^{\langle\mu} \sigma^{\nu\rangle \lambda}, \quad \mathcal{A}_{4}^{\mu \nu}=\sigma_{\lambda}^{\langle\mu} \Omega^{\nu\rangle \lambda}, \quad \mathcal{A}_{5}^{\mu \nu}=\Omega_{\lambda}^{\langle\mu} \Omega^{\nu\rangle \lambda} .
\end{aligned}
$$

Equation (34) can be recast as [33] $u^{\langle\rho} \nabla_{\rho} \sigma^{\mu \nu\rangle}+\frac{1}{2} \sigma^{\mu \nu}(\nabla \cdot u)$, which by using (33), reduces to the linear combination $\mathcal{A}_{1}^{\mu \nu}-\mathcal{A}_{2}^{\mu \nu}-(1 / 2) \mathcal{A}_{3}^{\mu \nu}-2 \mathcal{A}_{5}^{\mu \nu}$. The second-order dissipative part in the expansion of the stress-energy tensor (13) must therefore read

$$
\begin{aligned}
T_{(2)}^{\mu \nu}= & \eta \tau_{\Pi}\left[u^{\langle\rho} \nabla_{\rho} \sigma^{\mu \nu\rangle}+\frac{1}{2} \sigma^{\mu \nu}(\nabla \cdot u)\right] \\
& +\kappa\left(R^{\langle\mu \nu\rangle}-2 u_{\rho} u_{\tau} R^{\rho\langle\mu \nu\rangle \tau}\right) \\
& +\lambda_{1} \sigma_{\tau}^{\langle\mu} \sigma^{\nu\rangle \tau}+\lambda_{2} \sigma_{\tau}^{\langle\mu} \Omega^{\nu\rangle \tau}+\lambda_{3} \Omega_{\tau}^{\langle\mu} \Omega^{\nu\rangle \tau},
\end{aligned}
$$

where the $\kappa$ term contributes to the two-point Green function of the stress-energy tensor. The $\lambda_{i}$ terms are non-linear in velocity and the parameter $\tau_{\Pi}$ can be thought of as the relaxation time [33]. It is worth mentioning that the secondorder hydrodynamic transport coefficients $\kappa$ and $\lambda_{i}$ have been calculated from the leading-order AdS/CFT correlators at finite chemical potential and in the presence of fundamental matter $[33,38,39]$. They correspond to the black brane horizon response properties. Moreover, these coefficients have been derived for strongly coupled plasma described holographically by an Einstein-scalar action in the bulk, as functions of the temperature in lattice QCD thermodynamics [41]. Nonetheless, there is not a final word concerning the values of these parameters. For conformal theories at finite temperature, and zero chemical potential, they scale with suitable powers of the temperature and may depend on coupling constants and eventually on the rank of the gauge group [33,42]. In fact, there is an intrinsic ambiguity in second-order viscosity parameters in relativistic hydrodynamics [42]. For example, one of the coefficients can be calculated as $\lambda_{3}=-4 \lim _{\substack{k_{1} \rightarrow 0 \\ k_{2} \rightarrow 0}} \frac{\partial}{\partial_{k_{1}}} \frac{\partial}{\partial_{k_{2}}} \lim _{\substack{\omega_{1} \rightarrow 0 \\ \omega_{2} \rightarrow 0}} G^{x y|0 x| 0 y}$. The expressions thus obtained are very similar to those given in Refs. [33,39], namely

$$
\begin{aligned}
& \kappa=\frac{N_{c}^{2} \mathcal{T}^{2}}{8}(1-10 \gamma) \\
& \tau_{\Pi}=\frac{2-\ln 2}{2 \pi \mathcal{T}}+\frac{375 \gamma}{4 \pi \mathcal{T}}+\cdots \\
& \lambda_{1}=\frac{N_{c}^{2} \mathcal{T}^{2}}{16}(1+350 \gamma)
\end{aligned}
$$

$\lambda_{2}=-\frac{N_{c}^{2} \mathcal{T}^{2}}{16}[2 \ln 2+5(97+54 \ln 2) \gamma+\cdots]$

$\lambda_{3}=\frac{25 N_{c}^{2} \mathcal{T}^{2}}{2} \gamma+\cdots$

where $\gamma=\left(g^{2} N_{c}\right)^{-3 / 2} \zeta(3) / 8$ and $\mathcal{T}$ again denotes the system temperature. We naturally choose the latter to equal the black string Hawking temperature, given by Eqs. (13) and (21) in Ref. [29] for the CFM solutions I and II respectively, and depending on $M$ and $\beta$. The strong coupling limit $\gamma \rightarrow 0$ can also be obtained $[14,25]$. We emphasise that our results below are independent of changes in the above transport coefficients, at least for the parameters derived in Refs. [14,25,42].

Similar steps to the ones yielding Eq. (17) and the Kubo formula (21) can be repeated. If one now takes, as usual in the literature, $h_{12}=h_{12}(t, z)$, Eq. (36) yields

$$
\begin{aligned}
& T_{(1) 12}+T_{(2) 12} \\
& =-P h_{12}-\eta \dot{h}_{12}+\eta \tau_{\Pi} \ddot{h}_{12}-\frac{\kappa}{2}\left[\ddot{h}_{12}+\partial_{z}^{2} h_{12}\right],
\end{aligned}
$$

where dots denote time derivatives. The retarded Green function then reads

$G_{R}^{12 \mid 12}(\omega, k)=P-i \eta \omega+\eta \tau_{\Pi} \omega^{2}-\frac{\kappa}{2}\left[\omega^{2}+k^{2}\right]$,

which is the analogue of the Kubo formula with secondorder derivative terms taken into account. We then see the kinetic parameters $\tau_{\Pi}$ and $\kappa$ are the coefficients of the $\omega^{2}$ and $k^{2}$ terms in the low-momentum expansion of the retarded propagator.

Upon employing the above expressions and repeating the analysis of Sect. 3, one finds new bounds for the PPN parameter $\beta$, and preliminary results are shown in Fig. 2. All terms in Eq. (36) are included, with transport coefficients provided by Eqs. (37a)-(37e) correspond to the second term in the right hand side of Eq. (3). Clearly, the strong coupling limit $\gamma \rightarrow 0$ can be taken and Eq. (2) then appears as a particular case of Eq. (3). The plot in the left panel is for the CFM solution I and $M_{\circ}<M<10^{5} M_{\circ}$. For $M=M_{\circ}$, the PPN parameter must lie in $1.0021 \lesssim \beta \lesssim 1.0042$, whereas $1.0001 \lesssim \beta \lesssim 1.0013$ for a brane-world CFM I black hole having a mass equivalent to $10^{5}$ solar masses. Similarly, for the CFM case II, it is worth to mention that when $M=M_{\circ}$, the bound on the shear viscosity-to-entropy ratio yields $1.0014 \lesssim \beta \lesssim 1.0042$, and $1.0019 \lesssim \beta \lesssim 1.0044$ for $M=10^{5} M_{\circ}$. The numerical stability of these results has been checked by considering several different orders of magnitude for the t'Hooft coupling, and the differences were always found to be negligibly small. Moreover, refined expressions involving more transport coefficients, like in Ref. [41], could be used. However, such contributions in the viscosity-to-entropy ratio would amount at most to order $10^{-5}$ corrections in the bounds for $\beta$. Similarly, we tested other possible choices for the second- 

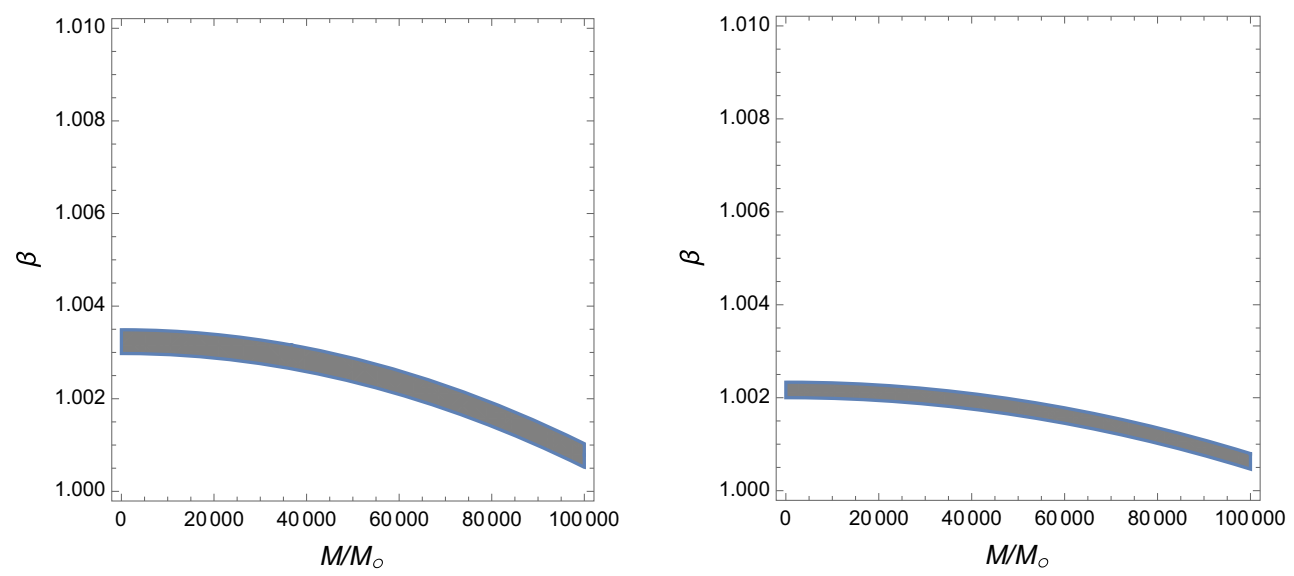

Fig. 2 Allowed range for the PPN parameter $\beta$ as a function of the mass $M$ (in Solar mass units), for CFM I (left panel) and CFM II (right panel) including second-order corrections

order viscosity parameters (37a)-(37e) and the differences with respect to our results were always found to be negligibly small. A comment is due about the relaxation time $\tau_{\Pi}$ in Eq. (37b), which turns out to affect the viscosity-toentropy ratio by a negligible order of $10^{-6}$. This transport coefficient, which appears in the first term on the r.h.s. of Eq. (36), varies with the inverse of the temperature, unlike the other coefficients in Eqs. (37a) and (37c)-(37e) which vary with the square of the temperature. Given the smallness of the black string temperature [43], it might seem that it would affect our results. However, this is not the case, since $\tau_{\Pi}$ multiplies a term that contains third-order covariant derivatives of the fluid velocity, and, by Eq. (17), is proportional to the third-order derivative of the perturbation $h_{12}$, which, in the regime of dual fluids under perturbations in the fluid/gravity setup, is identically zero. ${ }^{6}$ These preliminary results including second-order terms are thus fully compatible with the observational bound $|\beta-1| \lesssim 0.003$ provided by the perihelion shift [30]. Our aim here was to show the fluid/gravity correspondence can be a powerful method, by applying the KSS result. In particular, this correspondence allows one to obtain reasonable theoretical bounds for the already existing observational values of a post-Newtonian parameter. It is also worth emphasising that the CFM black brane should not satisfy any four-dimensional PPN bound a priori, since the black brane resides in five dimensions. It is, at least, an intriguing numerical coincidence that the PPN bound for the parameter of the CFM black brane matches the four-dimensional PPN bounds. Nevertheless, the main result here is that, since the PPN parameter is bound to be close to unity also in five dimensions, the CFM black branes must effectively reduce to their Schwarzschild counterparts. In fact, both CFM metrics (8) and (9) reduce to

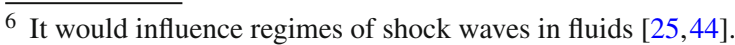

the Schwarzschild metric in the limit $\beta \rightarrow 1$. Here, we proved that the KSS result forces the CFM black branes to reduce to Schwarzschild black branes. For the boundary energy-momentum expansion (13), the first-order approximation (16) provides $|\beta-1| \lesssim 10^{-1}$, whereas the secondorder approximation (16) provides $|\beta-1| \lesssim 3 \times 10^{-2}$. When the higher-order term $\left\langle T_{(3)}^{\mu v}\right\rangle$ is included in Eq. (13), one finds $|\beta-1| \lesssim 10^{-4}$, suggesting that $\beta \rightarrow 1$ when sufficiently high-order terms $\left\langle T_{(n)}^{\mu v}\right\rangle$ are taken into account in Eq. (13). This is in agreement with the conjecture of Ref. [51] that the Schwarzschild black brane is the unique static blackhole solution of vacuum gravity with a negative cosmological constant.

\section{Discussion and outlook}

In this work, we derived the shear viscosity-to-entropy ratio $\eta / s$ as a function of the PPN parameter $\beta$ of candidate CFM black strings from the Green-Kubo formula in the context of the fluid/gravity correspondence. On assuming the KKS universal bound (2), we then determined the allowed range of $\beta$ for different black string mass. Our results show a possible slight dependence of $\beta$ on the mass in the range $M_{\circ}<M<10^{4} M_{\circ}$ in the linear-order hydrodynamic expansion (see Fig. 1). The non-linear hydrodynamical regime was just started to be investigated including second-order terms. In particular, when the terms displayed in Eq. (36) are included, with the transport coefficients (37a)(37e), the theoretical bounds are modified and appear in agreement the four-dimensional observational bounds (see Fig. 2). The discrepancy with experimental data shown in Fig. 1 could thus be simply related to the approximations employed in order to derive Eq. (32) from the Green-Kubo formula, for instance, the near horizon limit and the lin- 
earised hydrodynamics regime. Of course, this will be the subject of further investigations. We showed that the KSS result implies that the CFM black branes are, effectively, Schwarzschild black branes, when sufficiently high-order terms in the boundary energy-momentum tensor (13) are taken into account, in agreement with the conjectured unicity of the Schwarzschild black brane as a static black-hole solution of vacuum gravity with a negative cosmological constant [51].

As more possible developments, we recall that higher derivative gravity corrections have been considered [33,45]. The ratio of shear viscosity-to-entropy density indicates that the KSS bound (2) could then be violated, and the new lower bound $\eta / s \geq 4 / 25 \pi$ was proposed [21], based on causality of the dual field theory. In this context, it seems that such higher derivative gravity corrections make the CFM solutions inadequate as a black brane prescription. Indeed, from the bound $\eta / s \gtrsim 4 / 25 \pi$, the PPN parameter of these metrics would be $\beta \simeq 0.7432$, which contradicts the theoretical bound $\beta \geq 1$. A correction for $\eta / s$ was also given in Ref. [45] in the form of a linear combination of the coefficients $\alpha_{i}$ of the scalars $R, R_{\mu \nu} R^{\mu \nu}$ and $R_{\mu \nu \rho \sigma} R^{\mu \nu \rho \sigma}$ in the Gauss-Bonnet contribution to the action. More precisely, in the Gauss-Bonnet context, $\eta / s \simeq\left(1-8 \alpha_{3}\right) / 4 \pi$ and the KSS bound is violated for $\alpha_{3}>0$. However, since the CFM solutions are obtained with no Gauss-Bonnet terms, one can set $\alpha_{3}=0$ and there is no anisotropy, so that the KSS bound (32) is the only one that does hold for CFM metrics. The quark-gluon plasma produced in the ultra-relativistic collision of heavy ions at RHIC and LHC [46] is a strongly coupled fluid that is represented by the anisotropic evolution of the fluid in the initial stages before isotropisation. Recently, there has been some interest in modelling this anisotropy at strong coupling [47,48] and in studying how observables may be affected by it. Some of the studies include the computation of the shear viscosity-to-entropy density ratio $[49,50]$. Anisotropic scenarios can be further considered to include such possibilities.

Acknowledgments R.C. is partly supported by INFN grant FLAG. R.T.C. is supported by CAPES, PDSE and is grateful to R.C. for the hospitality. R.dR. is grateful to CNPq (Grants No. 303293/2015-2, and No. 473326/2013-2), and to FAPESP (Grant No. 2015/10270-0) for partial financial support. RdR thanks to Prof. J. Noronha for fruitful discussions.

Open Access This article is distributed under the terms of the Creative Commons Attribution 4.0 International License (http://creativecomm ons.org/licenses/by/4.0/), which permits unrestricted use, distribution, and reproduction in any medium, provided you give appropriate credit to the original author(s) and the source, provide a link to the Creative Commons license, and indicate if changes were made.

Funded by SCOAP . $^{3}$.

\section{References}

1. R. Maartens, K. Koyama, Brane-world gravity. Living Rev. Relativ. 13, 5 (2010)

2. I. Antoniadis, N. Arkani-Hamed, S. Dimopoulos, G.R. Dvali, New dimensions at a millimeter to a Fermi and superstrings at a TeV. Phys. Lett. B 436, 257 (1998)

3. I. Antoniadis, A possible new dimension at a few TeV. Phys. Lett. B 246, 377 (1990)

4. S. Dimopoulos, G.L. Landsberg, Black holes at the LHC. Phys. Rev. Lett. 87, 161602 (2001)

5. R. da Rocha, A. Piloyan, A.M. Kuerten, C.H. CoimbraAraujo, Casadio-Fabbri-Mazzacurati black strings and braneworld-induced quasars luminosity corrections. Class. Quant. Grav. 30, 045014 (2013)

6. S.S. Seahra, C. Clarkson, R. Maartens, Detecting extra dimensions with gravity wave spectroscopy: the black string brane-world. Phys. Rev. Lett. 94, 121302 (2005)

7. R. Casadio, J. Ovalle, R. da Rocha, Black strings from minimal geometric deformation in a variable tension brane-world. Class. Quant. Grav. 30, 175019 (2014)

8. D. Bazeia, J.M. Hoff da Silva, R. da Rocha, Regular bulk solutions and black strings from dynamical brane-worlds with variable tension. Phys. Rev. D 90, 047902 (2014)

9. Y. Bu, M. Lublinsky, Linearized fluid/gravity correspondence: from shear viscosity to all order hydrodynamics. JHEP 1411, 064 (2014)

10. J.M. Maldacena, The large $\mathrm{N}$ limit of superconformal field theories and supergravity. Adv. Theor. Math. Phys. 2, 231 (1998)

11. S.S. Gubser, I.R. Klebanov, A.M. Polyakov, Gauge theory correlators from non-critical string theory. Phys. Lett. B 428, 105 (1998)

12. O. Aharony, S.S. Gubser, J.M. Maldacena, H. Ooguri, Y. Oz, Large $\mathrm{N}$ field theories, string theory and gravity. Phys. Rep. 323, 183 (2000)

13. M. Natsuume, AdS/CFT duality user guide. Lect. Notes Phys. 903 (2015). arXiv:1409.3575 [hep-th]

14. G. Policastro, D.T. Son, A.O. Starinets, Shear viscosity of strongly coupled $\mathcal{N}=4$ supersymmetric Yang-Mills plasma. Phys. Rev. Lett. 87, 081601 (2001)

15. G. Policastro, D.T. Son, A.O. Starinets, From AdS/CFT correspondence to hydrodynamics. JHEP 0209, 043 (2002)

16. P. Kovtun, D.T. Son, A.O. Starinets, Holography and hydrodynamics: diffusion on stretched horizons. JHEP 0310, 064 (2003)

17. P. Kovtun, D.T. Son, A.O. Starinets, Viscosity in strongly interacting quantum field theories from black hole physics. Phys. Rev. Lett. 94, 111601 (2005)

18. A. Buchel, J.T. Liu, A.O. Starinets, Coupling constant dependence of the shear viscosity in $\mathcal{N}=4$ supersymmetric Yang-Mills theory. Nucl. Phys. B 707, 56 (2005)

19. R. Emparan, Absorption of scalars by extended objects. Nucl. Phys. B 516, 297 (1998)

20. S. Cremonini, The shear viscosity to entropy ratio: a status report. Mod. Phys. Lett. B 25, 1867 (2011)

21. R.G. Cai, Z.Y. Nie, N. Ohta, Y.W. Sun, Shear viscosity from GaussBonnet gravity with a dilaton coupling. Phys. Rev. D 79, 066004 (2009)

22. L. Lehner, F. Pretorius, Black strings, low viscosity fluids, and violation of cosmic censorship. Phys. Rev. Lett. 105, 101102 (2010)

23. K.M. O’Hara, S.L. Hemmer, M.E. Gehm, S.R. Granade, J.E. Thomas, Observation of a strongly-interacting degenerate Fermi gas of atoms. Science 298, 2179 (2002)

24. A. Cherman, T.D. Cohen, P.M. Hohler, A sticky business: the status of the conjectured viscosity/entropy density bound. JHEP $\mathbf{0 8 0 2}$, 026 (2008) 
25. S. Bhattacharyya, V.E. Hubeny, S. Minwalla, M. Rangamani, Nonlinear fluid dynamics from gravity. JHEP 0802, 045 (2008)

26. M. Sadeghi, S. Parvizi, Hydrodynamics of a black brane in GaussBonnet massive gravity. Class. Quant. Grav. 33, 035005 (2016)

27. R. Casadio, L. Mazzacurati, Bulk shape of brane world black holes. Mod. Phys. Lett. A 18, 651 (2003)

28. R. da Rocha, J.M. Hoff da Silva, Black string corrections in variable tension brane-world scenarios. Phys. Rev. D 85, 046009 (2012)

29. R. Casadio, A. Fabbri, L. Mazzacurati, New black holes in the brane world? Phys. Rev. D 65, 084040 (2002)

30. C.M. Will, The confrontation between general relativity and experiment. Living Rev. Relativ. 9, 3 (2006)

31. N. Dadhich, R. Maartens, P. Papadopoulos, V. Rezania, Black holes on the brane. Phys. Lett. B 487, 1 (2000)

32. R. Gregory, R. Laflamme, Black strings and p-branes are unstable. Phys. Rev. Lett. 70, 2837 (1993)

33. P. Arnold, D. Vaman, C. Wu, W. Xiao, Second order hydrodynamic coefficients from 3-point stress tensor correlators via AdS/CFT. JHEP 1110, 033 (2011)

34. D.T. Son, Hydrodynamics and gauge/gravity duality. Nucl. Phys. Proc. Suppl. 192, 113 (2009)

35. D.T. Son, A.O. Starinets, Viscosity, black holes, and quantum field theory. Annu. Rev. Nucl. Part. Sci. 57, 95 (2007)

36. J.M. Hoff da Silva, R. da Rocha, Effective monopoles within thick branes. Europhys. Lett. 100, 11001 (2012)

37. P. Kovtun, G.D. Moore, P. Romatschke, The stickiness of sound: an absolute lower limit on viscosity and the breakdown of second order relativistic hydrodynamics. Phys. Rev. D 84, 025006 (2011)

38. G.D. Moore, K.A. Sohrabi, Thermodynamical second-order hydrodynamic coefficients. JHEP 1211, 148 (2012)

39. S. Grozdanov, A.O. Starinets, On the universal identity in second order hydrodynamics. JHEP 1503, 007 (2015)

40. H. Nastase, Introduction to the AdS/CFT Correspondence (Cambridge University Press, Cambridge, 2015)
41. S.I. Finazzo, R. Rougemont, H. Marrochio, J. Noronha, Hydrodynamic transport coefficients for the non-conformal quark-gluon plasma from holography. JHEP 1502, 051 (2015)

42. Y. Nakayama, Intrinsic ambiguity in second order viscosity parameters in relativistic hydrodynamics. Int. J. Mod. Phys. A 27, 1250125 (2012)

43. E. Abdalla, B. Cuadros-Melgar, A.B. Pavan, C. Molina, Stability and thermodynamics of brane black holes. Nucl. Phys. B 752, 40 (2006)

44. G.T. Horowitz, N. Itzhaki, Black holes, shock waves, and causality in the AdS/CFT correspondence. JHEP 9902, 010 (1999)

45. M. Brigante, H. Liu, R.C. Myers, S. Shenker, S. Yaida, Viscosity bound violation in higher derivative gravity. Phys. Rev. D 77, 126006 (2008)

46. J. Adams et al. [STAR Collaboration], Experimental and theoretical challenges in the search for the quark gluon plasma: the STAR collaborations critical assessment of the evidence from RHIC collisions. Nucl. Phys. A 757, 102 (2005)

47. D. Mateos, D. Trancanelli, Thermodynamics and instabilities of a strongly coupled anisotropic plasma. JHEP 1107, 054 (2011)

48. R. Critelli, S.I. Finazzo, M.Zaniboni, J. Noronha, Anisotropic shear viscosity of a strongly coupled non-Abelian plasma from magnetic branes. Phys. Rev. D 90, 066006 (2014)

49. A. Rebhan, D. Steineder, Violation of the holographic viscosity bound in a strongly coupled anisotropic plasma. Phys. Rev. Lett. 108, 021601 (2012)

50. E. Megias, F. Pena-Benitez, Holographic gravitational anomaly in first and second order hydrodynamics. JHEP 1305, 115 (2013)

51. R. Emparan, H.S. Reall, Black holes in higher dimensions. Living Rev. Relativ. 11, 6 (2008) 